\title{
The Effect of the Corotation on the Radial Gradient of Metallicity of Spiral Galaxies
}

\author{
Sergio Scarano Jr $\mathbf{r}^{1,2}$ and Jacques R. D. Lépine ${ }^{1}$ \\ ${ }^{1}$ Instituto de Astronomia, Geofísica e Ciências Atmosféricas da Universidade de São Paulo, \\ CEP 05508-900 São Paulo, SP, Brazil \\ email: scarano@astro.iag.usp.br / jacques@astro.iag.usp.br \\ ${ }^{2}$ Southern Astrophysical Research Telescope (SOAR), Cerro Pachon, Chile \\ email: scaranojr@ctio.noao.edu
}

\begin{abstract}
The corotation radius in a spiral galaxy is the place where the spiral pattern speed has the same velocity of the rotation curve. By compiling results coming from the literature for 20 spiral galaxies we verified a strong correlation between the radius of the minima or inflections of the metallicity distribution and the corotation radius.
\end{abstract}

Keywords. galaxies: abundances, dynamics, spiral, corotation, metallicity gradient.

\section{Introduction}

The gradients of metallicity observed in disk galaxies provide important constraints to the models of the chemical evolution of these objects. It is frequently observed, in a first approximation, that spiral galaxies have a declining gradient of metallicity. However, it is not rare to observe galaxies with a clear change in the slope of their gradient of metallicity (eg. Zaritsky et al., 1994). Considering that spiral arms are the main contributors to the formation of massive stars, which in turn are responsible for the metal enrichment of the interstellar medium, Mishurov et al. (2002) proposed a simple model for the chemical evolution of spiral galaxies that effectively introduces the role of the spiral arms in the star formation rate. This model is able to explain the plateau observed in the metallicity distribution of our galaxy, which would be a consequence of the minimum expected in the metallicity distribution produced at corotation. In this work we compiled a set of spiral galaxies for which the rotation curves, the gradients of metallicity and the corotation radii (or the spiral pattern speeds) have been evaluated in the literature, to verify if the variations in their metallicity gradient are also related to the corotation.

\section{Sample and Data Analysis}

There are many works on gradients of metallicity and on the rotation curves of spiral galaxies, but the corotation radii or the spiral pattern speeds were only estimated for a few galaxies. The observational data for 20 spiral galaxies presented here are distributed over 108 references compiled and homogenized by Scarano Jr. (2008). We adopted the Oxygen abundances observed in $\mathrm{H}$ II regions to evaluate the metallicity distribution. Distances inside the plane of each galaxy were recalculated to a same reference base. All methods used to determine the corotation were considered equivalent. The changes in the slope of the metallicity distribution were calculated from a 4 th order polynomial fitted to the observational data, which is enough to reproduce the expected radial declining behavior of the metallicities and admits a minimum inside it. Minima and inflections were 
determined using the derivatives of the fitted curve. The uncertainties were calculated by propagating the errors in the fitted parameters.

\section{Results and Conclusions}

Plotting the minimum or the inflection radius $R_{d Z}$, calculated with the procedure described above, against the median corotation radius $R_{C R}$, determined from the literature for each galaxy, and doing the same thing with the angular velocity $\Omega_{d Z}$, associated to $R_{d Z}$, against the correspondent spiral pattern speed $\Omega_{p}$, we obtained the correlations shown in Fig. 1 for all galaxies in our sample.
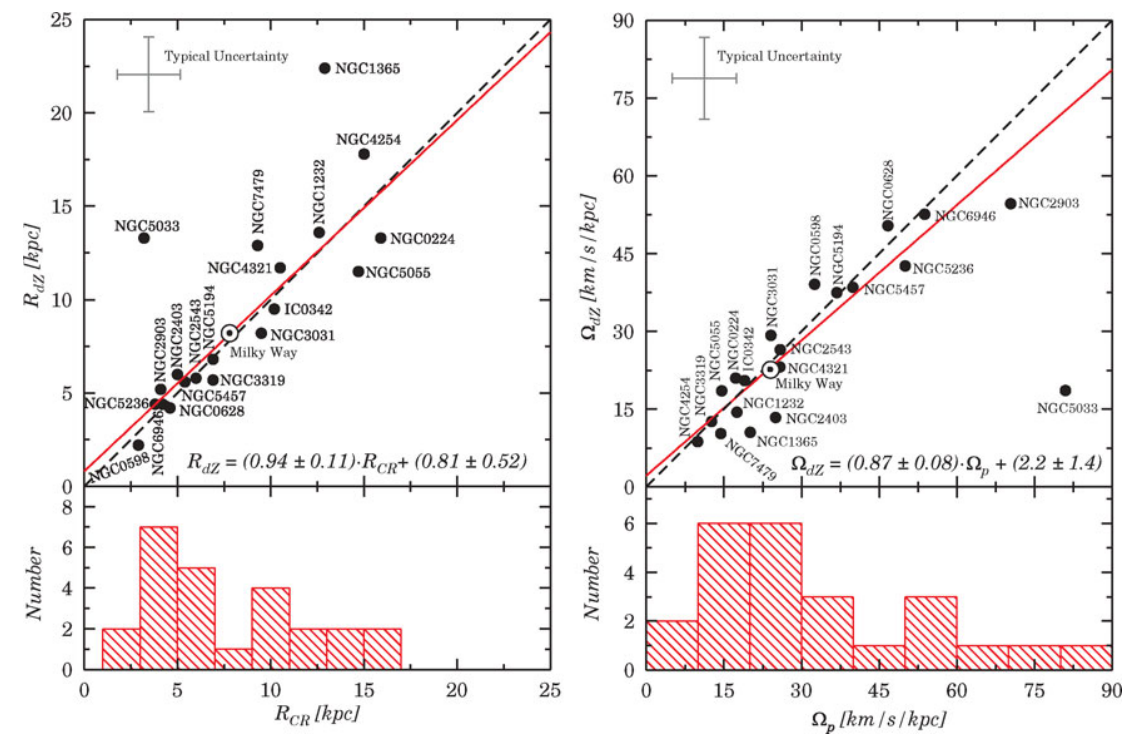

Figure 1. Correlation between the minima or inflection radii in the metallicity distribution and the corotation radii (left) and the equivalent graph for the angular velocities associated to breaks in the gradients of metallicity and the velocities of spiral pattern speed of all sampled galaxies (right). The Sun symbol represents the results for our galaxy. The histograms at the bottom of each graph show the statistics (number of points per bin) of the data.

In this figure the continuous lines are the linear regressions using robust statistics methods, which show the strong correlation between the radii of the breaks in the metallicity gradients and of corotation. The fitted lines are very near to the one-to-one correspondence represented by the dashed lines. Concerning other interesting relations discovered in the present study, the $\Omega_{p}$ distribution shows a peak around $20 \mathrm{~km} / \mathrm{s} / \mathrm{kpc}$, revealing that our galaxy is a typical one in this aspect. A surprising correlation between the corotation radius and the Elmegreen spiral arm class was also found, and when it is applied to our Galaxy, it results in a spiral pattern similar to that revealed by recent observations from the Spitzer telescope (Churchwell et al., 2009).

\section{References}

Churchwell, E. et al. 2009, PASP, 121, 213

Mishurov, Y. N., Lépine, J. R. D. \& Acharova, I. A. 2002, ApJ, 571L, 113M

Scarano Jr, S. 2008, PhD thesis, Universidade de Sao Paulo.

Zaritsky, D., Kennicutt, Jr., R. C., \& Huchra, J. P. 1994, ApJ, 420, $87 Z$ 